\title{
Study on Fusion of Terrestrial 3D Laser Point-clouds and Camera Image Data
}

\author{
Wenshu Lin, Yang Li, Jinzhuo Wu*, Shanshan Zhang and Yuan Meng \\ College of Engineering and Technology, Northeast Forestry University, Harbin \\ 150040, China \\ linwenshu@126.com,18845724597@163.com \\ *wujinzhuo1980@163.com,zss456zss@163.com,xfy0820@126.con
}

\begin{abstract}
Some point-cloud holes usually exist in the point-cloud data of trees acquired by a terrestrial 3D laser scanner. Such holes can affect the integrity of point-cloud data and subsequent $3 D$ reconstruction work. In order to solve the problems such as point-cloud holes or incomplete point-cloud data caused by the shield of obstacles or unable setup of $3 D$ laser scanner, this study focuses on the registration of point-cloud data and repairing the point-cloud holes aided by photogrammetry after analyzing the point-cloud data, and discusses the precise registration of point-cloud data based on ICP (Iterative Closest Point) algorithm and the fusion of scanned point-clouds with point-clouds generated by images. The purpose of this study lies on the registration of the target point clouds acquired by a terrestrial laser scanner with the point clouds generated from the entity image data, so as to ensure the integrity of the entity point-cloud data. Firstly, a terrestrial 3D laser scanner is used to acquire the point-cloud data of two Chinese pine (Pinus tabulaeformis) trees in the scanning region, and at the same time the algorithm of ICP is used to register the scanned point clouds. Then, in order to make up the pointcloud data holes caused by external objective factors, a digital camera is used to take pictures of the Chinese pine trees and acquire the image data that has high degree of overlap. The pairwise matching of homonymy feature points of the adjacent images taken on site is completed by using SIFT (Scale Invariant Feature Transform) image stitching algorithm, and then PMVS (patch-based multi-view stereo) algorithm is used to generate the $3 D$ point set of the two target Chinese pine trees. Finally, in the VC++ environment the ICP algorithm is used to fuse the point-cloud data obtained by $3 D$ laser scanner with the $3 D$ point set generated by PMVS algorithm. The results show that the mean square error for point-cloud registration and fusion are 0.0353733 and 0.0009226364 , respectively, which indicate that the effects of the registration and fusion are satisfied. This research can accurately and quickly finish the registration of the point-cloud data obtained in different ways, solve the defects of point-cloud holes caused by objective factors in complex forest environments such as trees blocked or site settings and other factors, and realize the acquirement of complete three-dimensional point cloud of trees, which has played a key role for subsequent three-dimensional reconstruction and parameters extraction of the trees.
\end{abstract}

Keywords: Point-cloud data; data registration; $3 D$ point set; fusion processing

\section{Introduction}

The geo-spatial information technology is stepping into a new period, and computer vision, computer graphics, and computer aided design have made rapid progress in recent years. The acquirement and analysis on the spatial data that can accurately describe 3D

* Corresponding Author 
spatial information have become the basis of geo-spatial information study. More specifically, the reconstruction of 3D entity model has become an important subject. A 3D model can not only provide real visual perception, but also contain highly precise spatial information of the targets, which is very helpful for the subsequent measurement and analysis. The 3D reconstruction of target scene requires true image data and 3D spatial data. Of which, the acquirement of image data is relatively easy and convenient. The widely used image acquisition devices include digital camera, video camera, image grabber, scanner, and so on. However, how to rapidly and efficiently acquire the 3D spatial information of the target scene in the real world is always the hot spot in the field of surveying and mapping.

Currently, the common methods used to acquire spatial 3D information include traditional geodetic surveying and engineering surveying based on distance measurement and angle measurement, close-range photogrammetry based on computer vision technology, and newly emerged 3D laser scanning methods based on active measurement. The traditional way of obtaining 3D data not only consumes much time, but also has low sampling density. The acquired data is unable to describe the spatial object accurately and completely. In addition, the spatial objects and their surroundings are usually complicated, which increases the difficulty in acquiring the corresponding spatial data accurately and comprehensively. The emergence of terrestrial 3D laser scanning technology provides us with a nondestructive 3D measurement means with high resolution, which can make up the shortcoming of traditional measurement means. The technology can be applied in many complex operation environments, which can greatly increase the measurement efficiency, reduce labor input, and save plenty of time. Compared to the traditional means, this technology is rapid, highly efficient, high density, precise, non-contact, active, high in automatic level, and easy to operate. Therefore, 3D laser scanning technology can be widely applied in many fields such as topographical survey, mining survey, urban modeling, building construction and deformation detection, reverse engineering, etc.

Even though 3D laser scanning technology as an emerging measurement means has many advantages compared to traditional measurement means, in the process of operation, due to the limitations of measuring equipment and environment, the point-cloud data from each measurement only covers most of the surface of the scanned object, and the complete point-cloud data can only be acquired through multi-station measurements. Therefore, it is necessary to stitch and register such local point-cloud data. Efficient and accurate registration can provide good fundamental data for subsequent point-cloud 3D modeling. Meanwhile, when 3D laser scanning technology is applied in forestry, it is very common that the target trees were shaded by other trees due to the complex environment. Thus, the point-cloud data acquired from terrestrial 3D laser scanning often contains some areas that are impossible to be measured, resulting in point-cloud holes and leading to the loss of some local regional information. These holes can prevent complete visualization of the 3D model of the scanned object and can also affect the parameters retrieval after modeling. Since close-range image acquisition is relatively easy with high flexibility and cost efficient, the scanning staff can use a digital camera to take supplementary pictures from different angles. In this way, the advantages of the two technologies can be used sufficiently to obtain a whole picture of the scanned object. However, multi-source data fusion has been a difficult topic in the field of data processing. What we need to do is to overcome the problem by looking for the homonymy features between the two types of data and using the information of the overlapped parts to merge the two types of data in a unified coordinate system and align all elements geometrically through registration operation.

So far, several studies have been conducted on the fusion of image and point-cloud data at home and abroad. For example, Baltsavias summarized the advantages and disadvantages of laser scanning and photogrammetrical techniques in terms of the sensors 
used, data acquisition conditions, image formation principle, precision, etc. and demonstrated the possibility of combining the two techniques as well as their promising application prospect[1]. Habib et al. aligned the image coordinates with laser scanning coordinates by taking the laser scanner's feature information locations such as points and lines as the image-oriented standard [2-3]. Meanwhile, Habib applied iterative Hough transform and voting algorithm to make registration of magnetic resonance image with laser point-clouds on basis of a curved surface as the basic element and obtained relatively high precision [4]. Gonzalez et al. developed an effective and reliable fusion method for point-clouds and image. The point-clouds were from a terrestrial 3D laser scanner and the image was from a non-professional camera. They also provided their open source coding tools "USAlign", which is useful for others to take reference to. The essence of the method is still to transform point-clouds into intensity image to meet the need of texture mapping [5]. In addition, Guarnieri et al. combined laser scanning technology and aerial photogrammetrical technology for reconstruction of historic buildings [6]. Roux combined 3D laser scanning and aerial photogrammetry and used simplex matching strategy to make registration of point-clouds with image and rebuilt a 3D model [7]. Faugeras and Heber applied the concept of quaternary array in the registration of point-clouds and proposed a key method for complex registration problem, that is the registration method of point set to point set [8]. Horn, Stein and Medioni also used the method to complete point-cloud registration $[9,10]$. The method can directly get the transform relationship between the two point sets without estimating the initial location of the unregistered point-sets, which is easy to use, however, there are also some other shortcomings such as lots of field work, not suitable for all scenes, and low registration precision. Due to the limitation of the method, another type of registration method was put forward, that is the registration method based on the internal features of point-clouds. Currently, the most commonly used and studied algorithm is ICP (Iterative Closest Point). This algorithm was continuously developed and was demonstrated as one of the most efficient registration methods[11]. Since there are still some problems in the algorithm such as obtaining local optimum and lower registration efficiency, many scholars have made modifications on the algorithm. Chen and Medioni, Grant et al. used the point to point iterative method without knowing the relationship between the points and proved that the method had better convergence effect [12-13]. In consideration of the registration efficiency, Eo et al. firstly performed registration based on point-cloud density image and RANSAC algorithm and then used ICP algorithm to perform registration and their results showed that the running speed of the algorithm was significantly improved [14]. Senin noticed the difference of point-clouds obtained by different sensors in terms of point-cloud density, precision and spatial structure. He used fitting interpolation to increase the volume of point-cloud and then used ICP to perform registration. However, the interpolation precision cannot be effectively controlled [15]. In China, Dr. Li retrieved roads through fusion of remote sensing image gray-scale value, point-cloud intensity, and degree of dispersion, but the specific ratios of distribution of the three factors were not provided. In some areas where point-clouds were distorted, the results were not good enough [16]. Tang combined 3D laser scanning and digital photogrammetry to retrieve the spatial entity of a single tree [17]. Dr. Deng at Wuhan University proposed a method to make registration by combining optical image with point-cloud intensity image [18]. It is noted that for terrestrial laser scanning the intensity of the point-clouds can be significantly affected by many other factors. Even though the intensity image can be used to extract the features and the third dimension was added, it is only valid for the specific object and has limitations to the target object under study [19].

In summary, the currently existing point-cloud data fusion methods mainly focus on the retrieval of common geometrical features of point-clouds and image by way of collinearity equations on basis of central projection principle. As a matter of fact, the method is still a registration of $2 \mathrm{D}$ with $3 \mathrm{D}$, which has relatively low accuracy and a lot of 
restrictions. The purpose of this study is to obtain a multi-view point-cloud data of two target Chinese pine trees (Pinus tabulaeformis Carr) by a terrestrial 3D laser scanner and use ICP algorithm to register the point-clouds of the scanned trees. In consideration of the point-clouds holes caused by external objective factors, a non-professional camera is used to acquire the additional image data. The pairwise matching of homonymy feature points of the adjacent images taken on site is completed by using SIFT (Scale Invariant Feature Transform) image stitching algorithm, and then PMVS (patch-based multi-view stereo) algorithm is used to generate the 3D point set of the two target Chinese pine trees. Finally, in the $\mathrm{VC}++$ environment the ICP algorithm is used to fuse the point-cloud data obtained by 3D laser scanner and 3D point set generated by PMVS algorithm, so as to acquire the complete point-cloud data of the trees.

\section{Data Acquisition and Denoising}

\subsection{Profile of the Study Area}

The study area is located at Beijing Olympic Forest Park with geographical coordinates of $40^{\circ} 01^{\prime} 3.00$ " $\mathrm{N}$ and $116^{\circ} 23^{\prime} 2.98^{\prime \prime} \mathrm{E}$. The park is divided into the northern part and the southern part by the 5th Beltway. The northern park is selected as the site of our experiment for measurement and scanning. From the geomorphic view, the Olympic Forest Park belongs to alluvial plain in geological structure. In terms of climate features, the Olympic Forest Park is influenced by temperate monsoon climate, with four distinct seasons. Rainfall is mostly in spring and summer and the average annual temperature is 12.2 ${ }^{\circ} \mathrm{C}$ in 2015 . There are more than one hundred plant varieties in the park, including trees, shrubs and grass, such as Mongolian Scotch pine (Pinus sylvestnis), Chinese pine (Pinus tabulaeformis) and other trees, purple lilac (Syringa oblata), yellow thorn plum (Rosa xanthina) and other shrubs, which are very popular in northern China.

\subsection{Acquisition of Point-Cloud Data}

Two typical planted Chinese pine (Pinus tabulaeformis) trees were selected for scanning in the study area, and the scanning was conducted in April 2016. Firstly, the locations for various measurement stations and auxiliary scanning equipment are designed according to the size and shape of the selected Chinese Pine trees. To obtain a $360^{\circ}$ panorama view of the Chinese pine tree, a 3D laser scanner of FARO Laser Scanner Focus3D X 330 is used to scan the tree from three measurement stations that are erected around the sample tree to form an equilateral triangle on the drawing, as shown in Figure 1. Included in the auxiliary equipment are reference balls and target paper. The reference ball can provide a reference point for data registration, and the target paper can provide the location of the Chinese pine tree and facilitate subsequent point-cloud data processing. It is noted that at least three control targets should be seen in each measurement station. The locations of the control targets should maintain an appropriate distance from the trees. If the distance is too close or too far, the subsequent stitching precision will be affected. The targets should not be arranged on the same straight line and a certain height fall should be maintained. The 3D laser scanner is adjusted after its erection before the power of the scanner is turned on. The parameters of the scanner should be set up to suit the environment for automatic scanning on the Chinese pine trees. 


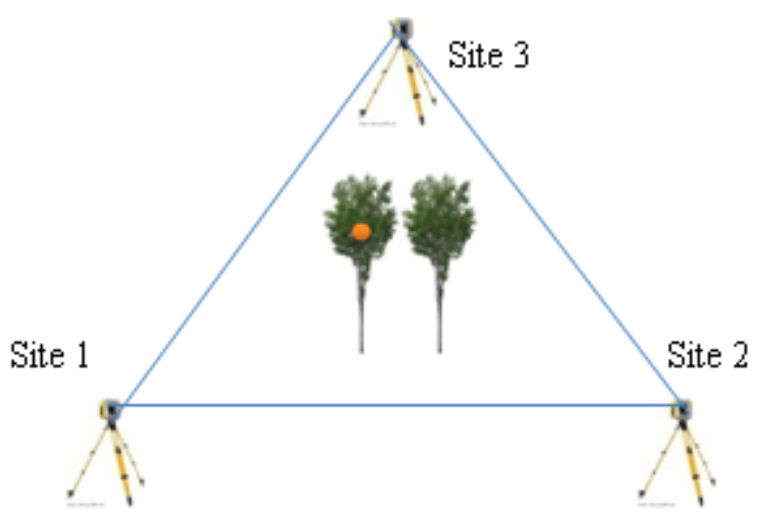

Figure 1. Schematic Diagram of 3D Laser Scanners

\subsection{Acquisition of Image Data}

Due to the influence of other trees, the point clouds of the scanned object cannot be displayed completely. To fill these point-cloud holes, the scanned object must be further scanned. The remedy method used in this study is to use a non-professional Canon EOS 500D video camera to take ominidirectional pictures of the Chinese pine around the tree on the scene of the acquired point-cloud data by terrestrial 3D laser scanner. The obtained image resolution is $3,456 \times 2,304$ pixels. At the same time, to increase the quantity of homonymy points between pictures, the degree of overlap of adjacent images should be about $70-80 \%$, so that the captured image data from the camera can meet the needs for 3D point set reconstruction.

\subsection{Denoising of Point-Cloud Data}

The process of data acquisition by terrestrial 3D laser scanning system can be affected by various factors, which will unavoidably introduce some noise to the point-cloud data acquired. Such factors include surface roughness and texture of trees, surroundings such as dust, wind and sheltering materials, precision of instruments, vibration, etc. Therefore, it is necessary to make denoising filtering process for point-cloud data from the scanned Chinese pine trees. The specific procedure is to import point-cloud data to the software Geomagic Studio 2012. The point-cloud data are rotated and scaled, and manual interpretation is made to find out and eject any significant noise points. Then, Geomagic Studio is used to preset a certain sensitive factor to eject any external isolated points. The next step is to set a certain deviation limit to eliminate noise, so that the points are lined up in a smoother way. Finally, further manual refining is carried out to the point-cloud data to fulfill optimization of the point-cloud data.

\section{Research Methods}

\subsection{Point-Cloud Registration}

\subsubsection{Rigid-Body Transformation}

In order to obtain the required three rotation angles and three translational parameters in registration, the rigid-body transformation is needed, and then the ICP algorithm can be applied for registration of point clouds of scanned trees. The two target trees are scanned respectively from station 1, station 2 and station 3, as shown in Figure 1. Assuming their respective scanning coordinate systems are $a_{1}, a_{2}$ and $a_{3}$, respectively, with their corresponding point-cloud data being point clouds 1 , point clouds 2 and point clouds 3 . By finding the rigid-body transformation from coordinate system $a_{1}$ to coordinate system 
$a_{2}$, we can further obtain major parameters of rotation matrix $R$ and translation vector $T$, which in turn can help us to find the coordinate transformation parameters between the adjacent stations. If both of the scanners at two different stations can see point $P$, and the point $P$ is any point on the surface of Chinese pine (Pinus tabulaeformis), and the coordinates of this point in the two point-cloud images can be assumed to be $P_{1}\left(x_{1}, y_{1}, z_{1}\right)^{T}$ and $P_{2}\left(x_{2}, y_{2}, z_{2}\right)^{T}$, where $P_{1}$ represents the coordinate of point $P$ in the first point clouds. Rotation and translation is made to convert the coordinate to the corresponding coordinate $P_{2}$ in the second point clouds. In the above-mentioned rigidbody transformation process, the 3D coordinates of any point $P$ in $a_{1}$ on the scene shall keep a relation as described below with its $3 \mathrm{D}$ coordinates in $a_{2}$ :

$$
\left(\begin{array}{l}
X_{p}{ }^{2} \\
Y_{p}{ }^{2} \\
Z_{p}{ }^{2}
\end{array}\right)=\left(\begin{array}{ccc}
\cos \varphi \cos \phi+\sin \varphi \sin \theta \sin \phi & -\cos \phi \sin \varphi+\sin \theta \sin \phi \cos \varphi & \sin \phi \cos \theta \\
\cos \theta \sin \varphi & \cos \theta \cos \varphi & -\sin \theta \\
-\sin \phi \cos \varphi+\sin \theta \sin \phi \sin \varphi & \sin \phi \sin \varphi+\sin \theta \cos \phi \cos \varphi & \cos \phi \cos \theta
\end{array}\right)\left(\begin{array}{l}
X_{p}{ }^{1} \\
Y_{p}{ }^{1} \\
Z_{p}{ }^{1}
\end{array}\right)+\left(\begin{array}{l}
t_{x} \\
t_{y} \\
t_{z}
\end{array}\right)
$$

In Equation (1), the translation vector $\mathrm{T}=\left(\begin{array}{c}t_{x} \\ t_{y} \\ t_{z}\end{array}\right)$. The rotation matrix $R$ can be described by the rotation angles of the three axes in Cartesian coordinate system. Assuming the rotation angles around axis $X$, axis $Y$ and axis $\mathrm{Z}$ are $\theta,{ }^{\phi}$, and $\varphi$, respectively, the rotation matrix $R$ is expressed as:

$$
R=\left(\begin{array}{lcc}
\cos \varphi \cos \phi+\sin \varphi \sin \theta \sin \phi & -\cos \phi \sin \varphi+\sin \theta \sin \phi \cos \varphi & \sin \phi \cos \theta \\
\cos \theta \sin \varphi & \cos \theta \cos \varphi & -\sin \theta \\
-\sin \phi \cos \varphi+\sin \theta \sin \phi \sin \varphi \sin \phi \sin \varphi+\sin \theta \cos \phi \cos \varphi & \cos \phi \cos \theta
\end{array}\right)
$$

\subsubsection{Parameter Initialization}

The point cloud set $Q$ of the trees acquired by 3D laser scanning contains $2,813,529$ points, i.e. $Q=\left(q_{1}, q_{2}, \cdots q_{2813529}\right)$. The rotation matrix, translation vector and other parameters in Equation (1) are used to make initialization. Assuming $k=0, P_{0}=P_{\left(P_{0}\right.}$ represents the initial position of the point set, $k$ is the cyclic iteration times), $R_{0}=I_{(I \text { is }}$ an identity matrix $), T_{0}=(0,0,0)^{T}$.

\subsubsection{Search for the Closest Point}

After parameter initialization, the closest point can be searched for. Firstly, the distances of each point $P_{k}=\left\{p_{i, k}\right\}$ in point set $P$ to each point in another point set $Q$ are calculated, and then they are replaced with the squared Euclidean distances $d(p, q)=\|p-q\|^{2}$, so as to find out the closest point $Y_{k}=\left\{y_{i, k}\right\}$. We define the function $e\left(R_{k}, T_{k}\right)$ of $R_{k}$ and $T_{k}$ as the MSE (mean square error) of the registration of the point pair $\left(p_{i, 0}, y_{i, k}\right)$. This study used the MSE to represent the accuracy of the point cloud registration, which can ensure the reliability of registration. Then obtain solutions for the 
rigid-body transformation parameters $\left(R_{k}, T_{k}\right)$, enabling $e\left(R_{k}, T_{k}\right)$ to have its minimum value, as shown in the equation below:

$$
\begin{gathered}
e\left(R_{k}, T_{k}\right)=\frac{1}{N_{p}} \sum_{i=1}^{N_{p}}\left\|\left(R_{k} p_{i, 0}+T_{k}\right)-y_{i, k}\right\|^{2} \\
e_{k}=\min _{R_{k}, T_{k}} e\left(R_{k}, T_{k}\right)
\end{gathered}
$$

On basis of the rigid-body transformation parameters obtained from the previous calculation step, the new coordinate positions of the reference point set $\mathrm{P}$ can be obtained, that is $P_{k+1}=\left\{P_{i, k+1}\right\}: P_{i, k+1}=R_{k} P_{i, 0}+T_{k}$. Make iteration calculation of the aforesaid step. Iteration termination conditions are preset, when the number of iterations $k$ reaches the preset maximum number of iteration times or the error has converged to a given threshold $\tau$, i.e. $e_{k-1}-e_{k}<\tau$. At this time, the rotation matrix $R=R_{k}$, and the translation matrix $T=T_{k}$, otherwise the next iteration shall follow. The specific registration flowchart is shown in Figure 2.

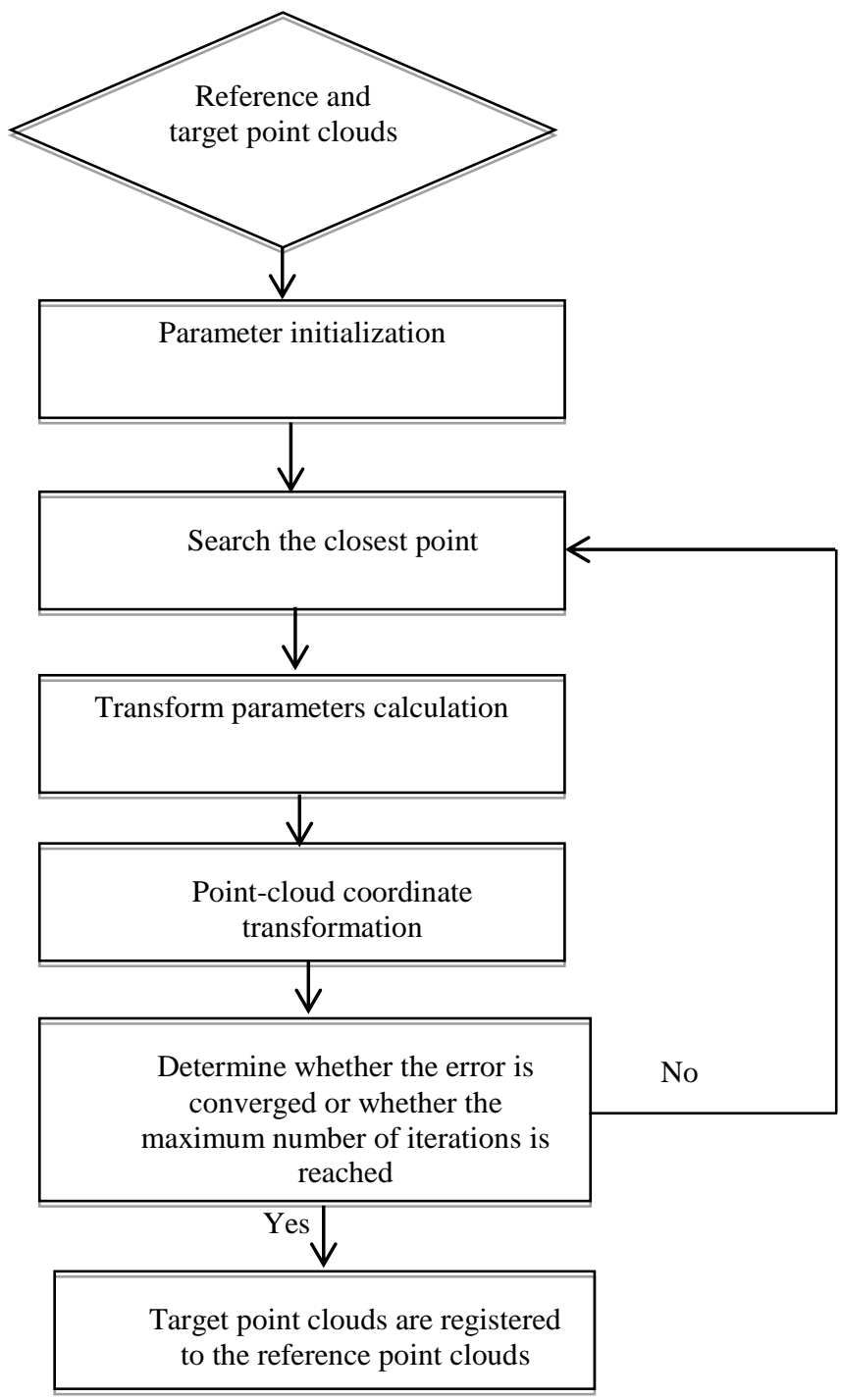

Figure 2. Registration Flowchart 


\subsection{Reconstruction of 3D Point Set of Image}

Pairwise matching of homonymy feature points of the adjacent images taken on site is conducted by SIFT image stitching algorithm, and then PMVS algorithm is used to obtain the $3 \mathrm{D}$ point set of the two target Chinese pine trees.

\subsubsection{Matching of Homonymy Feature Points}

(1) Detection of point-cloud feature points of trees in scale-space

To enable the scale invariance of point-cloud features, detection of the feature points of the point clouds is usually achieved in a multi-scale space. Gaussian convolution kernel is used to make scaling to the initial camera image, to obtain the scale-space presentation sequence of the image under multi-scales. Then the scale-space features are retrieved from these sequences. The images taken under different scale-space can be presented with Gaussian kernel convolution formula:

$$
L(x, y, \sigma)=G(x, y, \sigma) * I(x, y)
$$

where $L$ is the scale-space of the image; $(x, y)$ is the coordinates of image pixel; ${ }^{\sigma}$ is the scale coordinate. Continuous smoothing and down-sampling are made to the image to be processed with Gaussian kernel function at different scales. Then subtraction operation is made between two Gaussian images that have adjacent scales among the 150 image intervals to obtain DoG images. The same operation is carried out to all points within the image scale-space, and each point is compared with its adjacent points. If the sampling point is a local extreme point (maximum gray scale value or minimum gray scale value), it is considered a candidate feature point. In this way, we can guarantee that all candidate feature points are extreme points both in scale-space and 2D image space, and so that the locations of the trees' point-cloud feature points and their corresponding scales can be initially identified.

(2) Filtering and precise positioning of trees' point-cloud feature points

After the extreme points are identified in the first step, information such as the locations and scales of the feature points shall be determined. Not all these extreme points found in the previous step are stable extreme points. They may contain some noisesensitive low-contrast points and unstable edge-response points. Such two types of points can affect the matching precision. Therefore, they need to be eliminated before feature point matching. The Taylor series is used to expand the scale-space equation $\mathrm{D}(x, y, \sigma)$, as follows:

$$
D(X)=D+\frac{\partial D^{T}}{\partial x} X+\frac{1}{2} X^{T} \frac{\partial^{2} D}{\partial x^{2}} X
$$

$$
\begin{aligned}
& \text { where } X=(x, y, \sigma)^{T} \\
& \frac{\partial D}{\partial x}=\left[\begin{array}{l}
\frac{\partial D}{\partial x} \\
\frac{\partial D}{\partial y} \\
\frac{\partial D}{\partial \sigma}
\end{array}\right], \frac{\partial^{2} D}{\partial x^{2}}=\left[\begin{array}{l}
\frac{\partial^{2} D}{\partial x^{2}} \frac{\partial^{2} D}{\partial x y} \frac{\partial^{2} D}{\partial \sigma} \\
\frac{\partial^{2} D}{\partial y x} \frac{\partial^{2} D}{\partial y^{2}} \frac{\partial^{2} D}{\partial y \sigma} \\
\frac{\partial^{2} D}{\partial \sigma x} \frac{\partial^{2} D}{\partial \sigma y} \frac{\partial^{2} D}{\partial \sigma^{2}}
\end{array}\right]
\end{aligned}
$$


The first and second order derivatives in Equation (6) are calculated by differential approximation in the nearby area. Take derivative on Equation (6) and make it equal to zero to get the precise extreme-value location $\hat{X}_{\text {of }}$ the feature point:

$$
\hat{X}=-\frac{\partial^{2} D^{-1}}{\partial x^{2}} \frac{\partial D}{\partial x}
$$

(1)Removal of low-contrast points: Taking Equation (7) into Equation (6), we can get:

$$
D(\hat{X})=D+\frac{1}{2} \frac{\partial D^{T}}{\partial x}
$$

If $|D(\hat{X})| \geq 0.03$

, the feature point is retained, otherwise it is rejected.

(2) Removal of unstable edge-response points: A $2 \times 2$ Hessian matrix, denoted as $\mathrm{H}$, as shown in Equation (9), is used to calculate the principal curvatures. Let $\alpha$ and $\beta$ be the maximum and minimum feature values of $H$ respectively, as shown in Equation (10)., and $\gamma$ be their ratio, i.e. $\gamma=\frac{\alpha}{\beta}$

$$
H=\left[\begin{array}{l}
D_{x x} D_{x y} \\
D_{x y} D_{y y}
\end{array}\right]
$$

$$
\left\{\begin{array}{l}
\operatorname{Tr}(H)=D_{x x}+D_{y y}=\alpha+\beta \\
\operatorname{Det}(H)=D_{x x} D_{y y}-\left(D_{x y}\right)^{2}=\alpha \beta \\
\frac{\operatorname{Tr}(H)^{2}}{\operatorname{Det}(H)}=\frac{(\alpha+\beta)^{2}}{\alpha \beta}=\frac{(\gamma \beta+\beta)^{2}}{\gamma \beta^{2}}=\frac{(\gamma+1)^{2}}{\gamma}
\end{array}\right.
$$

If $\frac{\operatorname{Tr}(H)^{2}}{\operatorname{Det}(H)}<\frac{(\gamma+1)^{2}}{\gamma} \quad$ (generally $\gamma=10$ ), the feature point shall be retained, otherwise it should be rejected.

(3) Determination of the main direction of the feature point

The gradient direction distribution feature of the pixels in the area adjacent to the feature point is used to assign directional parameters for each feature point, so the SIFT operator has rotational invariance. The gradient value and direction of a specific feature point at coordinates $(x, y)$ are as follows respectively:

$$
\begin{aligned}
& m(x, y)=\sqrt{(L(x+1, y)-L(x-1, y))^{2}+(L(x, y+1)-L(x, y-1))^{2}} \\
& \theta(x, y)=\tan ^{-1}(L(x, y+1)-L(x, y-1)) /(L(x+1, y)-L(x-1, y))
\end{aligned}
$$

(4) Matching the feature point vector

After generating SIFT feature vectors from two images, the Euclidean distances of the point-cloud feature vectors are taken as the criterion for feature point similarity between 
the two images to get matched SIFT point pairs that meet the criterion. Take a feature point from an image and find out the first two feature points that have closest Euclidean distance to another image. If the closest distance divided by the second closest distance is less than a certain threshold, such point pair can be accepted. Finally, the image transformation parameters can be calculated according to SIFT matched point pairs obtained, and the stitching image can be achieved through image stitching fusion.

\subsubsection{Generation of 3D Point Set}

The PMVS algorithm is used to obtain a 3D point set. Firstly, detect the feature points in each image, and then match these feature points and recover their depth information on triangulation principle, so as to obtain the sparse 3D point clouds of the trees' surface feature points. The specific algorithm steps are as follows:

(1) Matching of feature points

Firstly, the corner features in each image are detected by feature detection operator (DoG operator) followed by seed patch extension.

(2) Seed patch extension

The purpose of seed patch extension is to enable the reconstructed patch set to fully cover the visible surface of the trees. The criterion is each image block $C(x, y)$ contains projection of at least one patch, so as to obtain complete information of tree surface. To a patch $p$, a new patch $p^{\prime}$ is obtained in its adjacent image block set $C(p)$ through extension.

\subsection{Fusion of Point-Cloud and Image Point Set}

The txt-format data acquired by terrestrial laser scanning and the ply-format data acquired from pictures shall be converted into unified .pcd-format point-cloud files. In $\mathrm{VC}++$ environment, the ICP algorithm is used to make registration of the acquired in-situ Chinese pine tree point-cloud data by 3D laser scanner with the 3D point set obtained by reconstruction of 3D Chinese pine tree image so as to offset the point-cloud holes and other defects caused by some objective factors, such as blocked trees and site setting reasons, and acquire complete point cloud of the Chinese pine tree as an entity.

\section{Results and Analysis}

\subsection{Registration Results}

The registration result of the scanned Chinese pine tree's point-cloud data by ICP algorithm in $\mathrm{VC}++$ environment is shown in Figure 3. The green point clouds are the reference point clouds and the red point clouds are the target point clouds. The target point clouds are registered to the reference point clouds through rotation matrix and translation vector. The generated yellow point-cloud set is the result of registration of the red point-cloud set to the green point-cloud set. As can be seen from the figure, the effect of point-cloud registration by ICP algorithm is good, and the two point sets fit almost perfectly. The rotation matrix $R$ and translation vector $T$ are expressed as follows respectively:

$$
R=\left(\begin{array}{lll}
0.999835 & 0.0182372 & -0.000151845 \\
-0.0182347 & 0.999516 & -0.0252435 \\
-0.00030903 & 0.025242 & 0.999683
\end{array}\right) T=\left(\begin{array}{l}
-0.293062 \\
1.67915 \\
-0.152931
\end{array}\right)
$$

The MSE of the point clouds registration is 0.0353733 , which illustrates that the registration accuracy is high. 


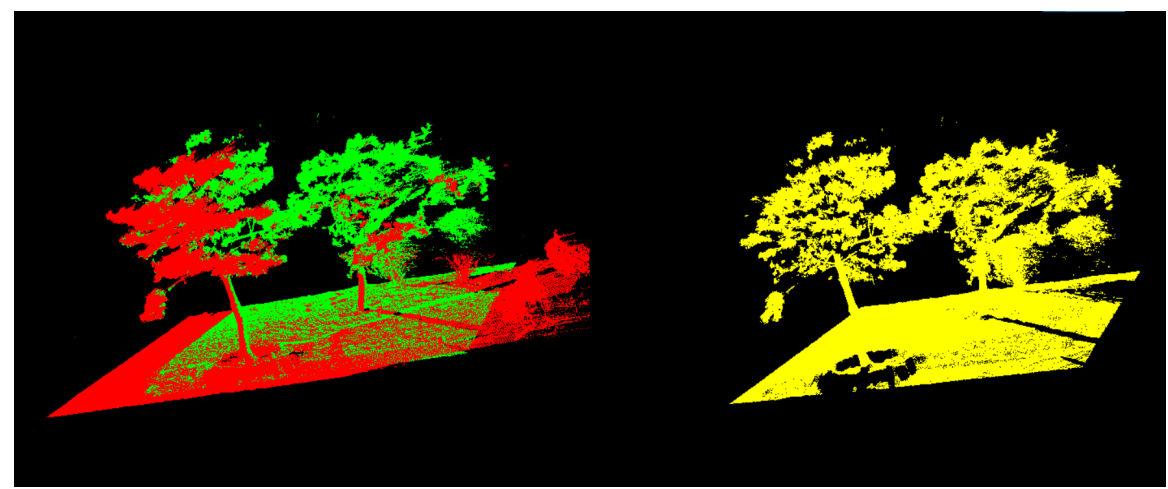

Figure 3. Registration in VC++ Environment

In order to see the registration effect more clearly, the generated registration results are displayed in Geomagic Studio 2012 software, as shown in Figure 4 (a). To verify the accuracy of the registration method used in this study, registration is also made directly to the originally obtained point-cloud data with the commercial software Realworks and the results are also displayed in the software Geomagic Studio 2012, as shown in Figure 4 (b). Based on the comparison in Figure 4, we can see that the shapes of the two Chinese pine trees are similar, thus the proposed registration algorithm in this paper is feasible. However, in terms of the completeness of the two trees displayed, some missing pointcloud data have caused insufficient completeness of the trees. Therefore, it is necessary to conduct some remedy work on the processed point-cloud data.

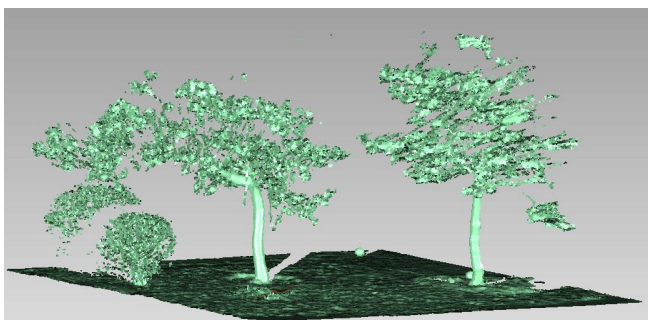

(a) ICP Algorithm Registration

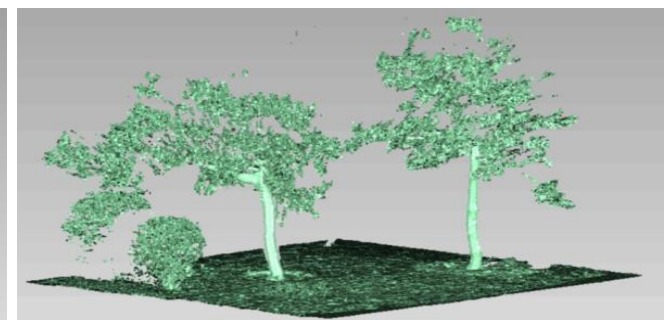

(b) Result of Software Registration

Figure 4. Results of Registration

\subsection{D Point Set}

In this study, the on-the-spot pictures of the scanned Chinese pine trees are obtained, and a total of 150 images are taken. Especially, intensive picture-taking is arranged in the places where point-cloud data holes exist after registration. By using the SIFT image stitching algorithm and multi-view stereo algorithm, the 3D point set $Q$ of the Chinese pine trees generated from processing of the on-the-spot images (Figure 5) contains 318,086 points, $Q=\left(Q_{1}, Q_{2}, \cdots, Q_{318086}\right)$, as shown in Figure 6:. 


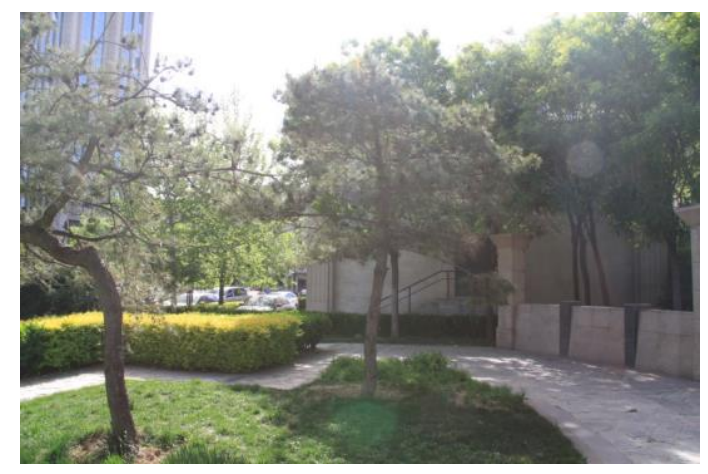

Figure 5. On-the-Spot Image

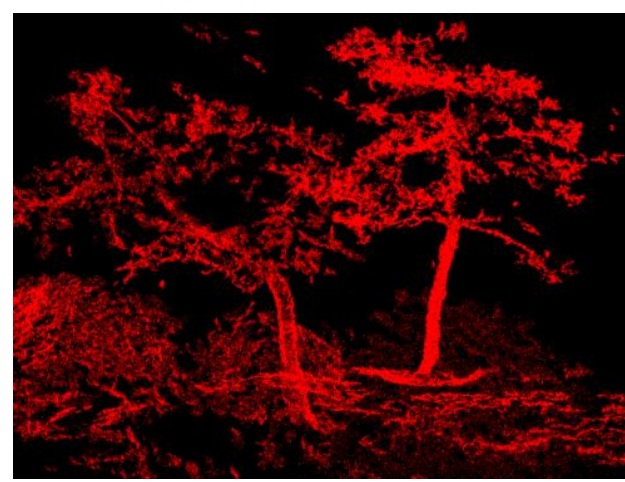

Figure 6. 3D Point Set

\subsection{Fusion of Point Clouds and Images}

After performing fusion on the registered scanned point-cloud data and the 3D image point clouds, the effect of point-cloud generation figure in Geomagic Studio 2012 is shown in Figure 7. The rotation matrix $R$ and translation vector $T$ are expressed as follows respectively:

$$
R=\left(\begin{array}{lrl}
-0.0133296 & -0.539683 & -0.84764 \\
0.650171 & 0.634899 & -0.417351 \\
0.759672 & -0.552853 & 0.342424
\end{array}\right), T=\left(\begin{array}{l}
65.8841 \\
22.2096 \\
15.3329
\end{array}\right) .
$$

The MSE of the point-clouds fusion is 0.0009226364 , which indicates that the fusion accuracy is very high. The number of points in the point-clouds before fusion is $2,813,529$, however, the number of points in the point-clouds after fusion increases to $3,131,615$, a significant increase. Through the contrast analysis with Figure 4, Figure 7 shows better results. The point-cloud data obtained after fusion not only increases pointcloud density, but also improve and repair the characteristics of scanned trees.

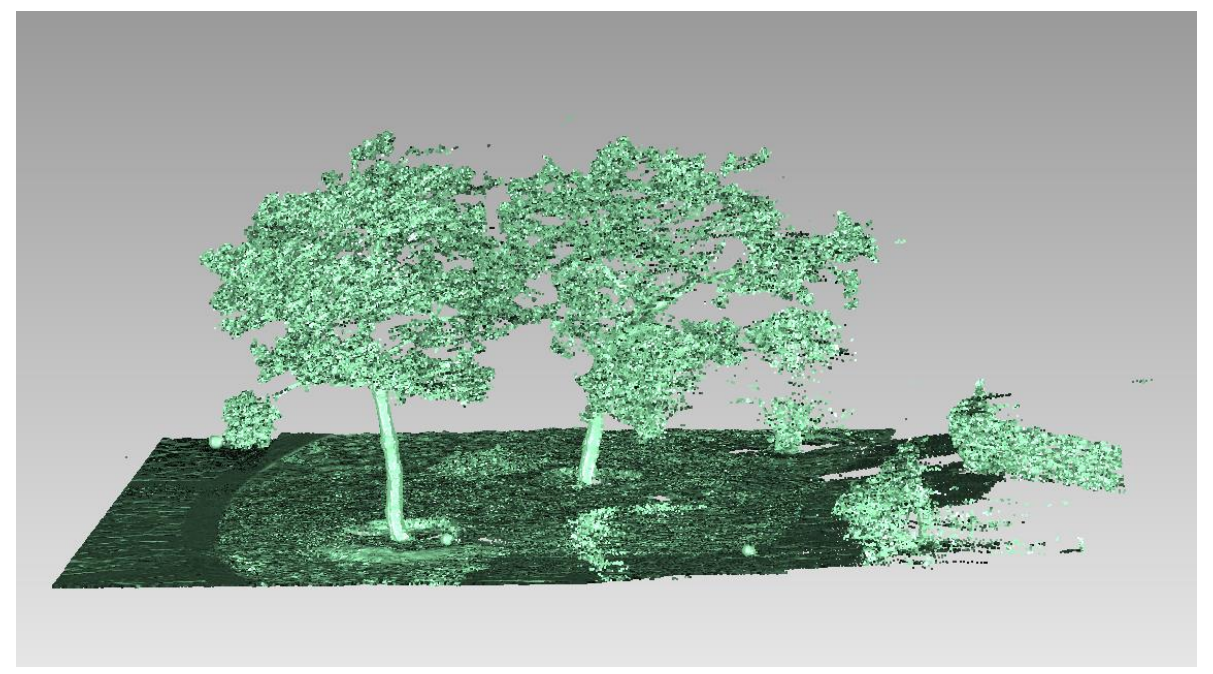

Figure 7. Image of Point-Cloud Fusion

In order to verify that the point-cloud image after fusion has been effectively repaired, the tree trunks and canopy part features are extracted from the images before and after image fusion, respectively. The results are shown in Figure 8 and Figure 9. It can be seen from Figure 8(a) that the point cloud image before fusion contains obvious defects of empty area, which will lead to the corresponding error in the subsequent processing. After 
fusion, as shown in Figure 8 (b), the point-cloud image is significantly improved and many empty areas are repaired, thus provides reliable data support for the subsequent point-cloud processing such as 3D modeling and tree parameters extraction.

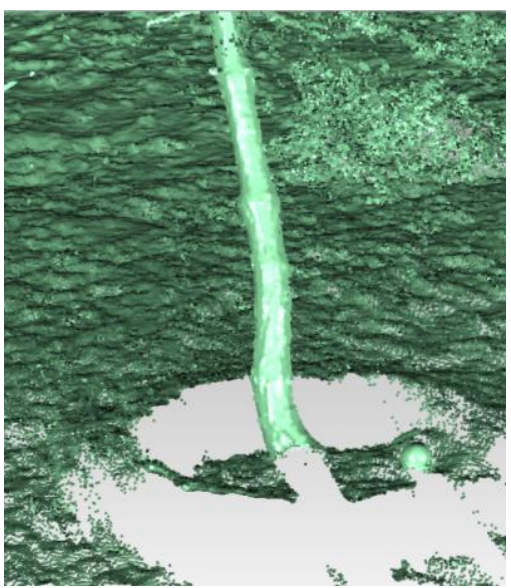

(a) Point Cloud Image Before Fusion

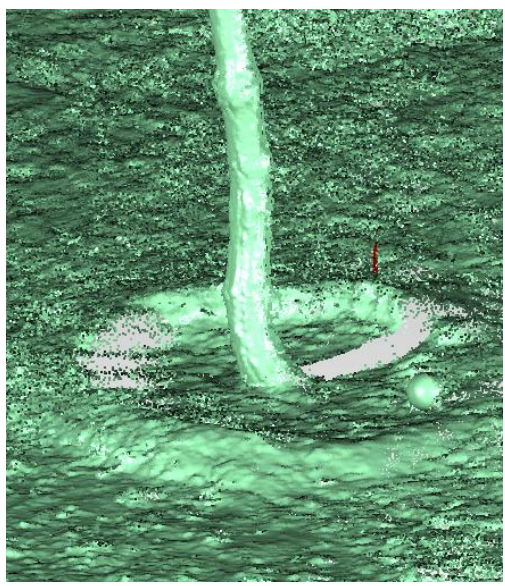

(b) Point Cloud Image After Fusion

Figure 8. Tree Trunk Point-Cloud Image Before and After Fusion

Similarly, as shown in Figure 9, after fusion the crown canopy image became better than that before fusion, and the characteristics of the crown canopy are effectively repaired to offset the problems of partial feature loss caused by objective factors such as terrain shade or scanning site settings, etc. In addition, the two kinds of point-cloud sets could be well overlapped in the process of fusion, making the precise combination between the laser point-cloud and image point-cloud come true, so as to ensure the wide application of ground three-dimensional laser scanning in complex forest environment.

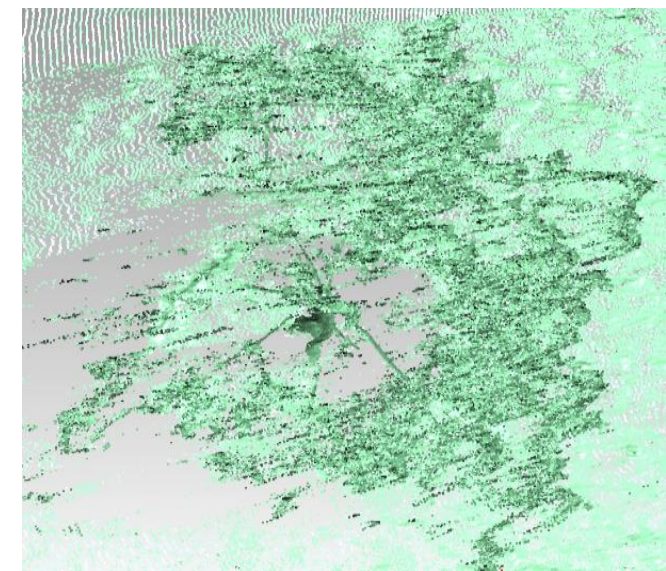

(a) Point-Cloud Image Before Fusion

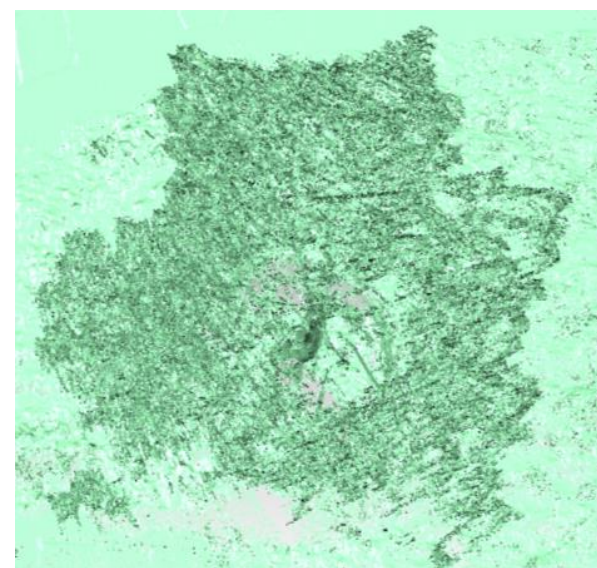

(b) Point-Cloud Image After Fusion

\section{Figure 9. Tree Crown Point-Cloud Images Before and After Fusion}

\section{Conclusion}

The study focuses on accurate registration of terrestrial 3D laser scanning point-cloud data and fusion to improve the incompleteness of acquired scanned point-cloud data in combination with close-range photogrammetrical techniques. Relatively complete 3D point-cloud data of trees are acquired through algorithm development. The results of the study are summarized as follows:

(1) The acquirement process of point-cloud data and a series of data preprocessing are introduced. Accurate registration of the trees' 3D point-cloud data is achieved by 
traditional ICP algorithm in VC++ environment on basis of different-view point-clouds and the mean square error of registration is 0.0353733 .

(2) The principle and procedure of generating point set from image is described in detail and the process of generating local point set of the object from image is realized. 3D point set of image data is generated on basis of the trees' pictures taken by a nonprofessional camera with SIFT image stitching algorithm and multi-view stereo algorithm;

(3) In VC++ environment, the point-cloud obtained by 3D laser scanner and the 3D point set obtained by image reconstruction are fused by using the ICP algorithm. The mean square error of fusion is 0.0009226364 , realizing precise fusion of point-cloud from $3 \mathrm{D}$ laser scanning and image. It is also shown from the tree trunk and tree crown pointclouds after fusion that the fusion results can effectively offset the defects of the original point-cloud and the complete entity point clouds of Chinese pine trees are acquired.

The results of this study will provide new ideas for fusion processing of point-clouds and images, broaden the application of ground three-dimensional laser scanning in complex forest environment, and lay a basis for subsequent authors to continue their study on improvement of ICP algorithm, 3D image reconstruction of trees and trees parameters extraction. Of course, to obtain high-quality complete point-cloud data, further studies are suggested in the following aspects: (1) Since the quality of point-clouds generated through image reconstruction is affected by image stitching processing accuracy and completeness, higher overlap degree of adjacent images of the on-the-spot object and higher stitching accuracy are required; and (2) To verify the feasibility of this method and show clear experiment results, the fusion object in our experiment is not a local portion of the entity but the whole object. To remedy the local defect holes of the object, the same way of operation can be carried out to solve the problem.

\section{Acknowledgments}

The authors would like to acknowledge the support of the Fundamental Research Funds for the Central Universities (2572016CB10), National Natural Science Foundation of China (31500587), Heilongjiang Province Postdoctoral Foundation (LBH-Z15007).

\section{References}

[1] E. P. Baltsavias, "A comparison between photogrammetry and laser scanning", ISPRS Journal of Photogrammetry and Remote Sensing, vol. 54, no. 2/3, (1999), pp. 83-94.

[2] A. F. Habib and M. S. Ghanma, "Using ground based laser scanners to establish the orientation of terrestrial imagery", The 6th Conference on Optical 3-D Measurements Techniques, Zurich, Switzerland, (2003).

[3] A. F. Habib, M. S. Ghanma, C. J. Kima and E.A. Mitishita, "Alternative approaches for utilizing lidar data as a source of control information for photogrammetric models", International Archives of XXth ISPRS Congress, Istanbul, Turkey, (2004).

[4] A. F. Habib, R T. Cheng, E. M. Kim, E. Mitishitta, R. Frayne and J.L. Ronsky, "Automatic surface matching for the registration of Lidar data and MR imagery", Electronic and Telecommunication Research Institute Journal, vol. 28, no. 2, (2006), pp. 162-174.

[5] A. D. Gonzalez, G. P. Rodriguez and L. J. Gomez, "An automatic procedure for co-registration of terrestrial laser scanners and digital cameras", ISPRS Journal of Photogrammetry and Remote Sensing, vol. 64, no. 3, (2009), pp. 308-316.

[6] A. Guarnieri, N. Milan and A. Vettore, "Monitoring of complex structure for structural control using terrestrial laser scanning (TLS) and photogrammetry", International Journal of Architectural Heritage, vol. 7, no. 1, (2013), pp. 54-67.

[7] M. Roux, "Registration of airborne laser data with one aerial image", International Archives of XXth ISPRS Congress, Istanbul, Turkey, (2004).

[8] . D. Faugeras and M. Hebert, "The representation, recognition and locating of 3D objects", International Journal of Robotic Research, vol. 5, no. 3, (1986), pp. 27-52.

[9] B. K. P. Horn, "Closed-form solution of absolute orientation using unit quaternions", Journal of the Optical Society of America, vol. 4, no. 4, (1987), pp. 629-642.

[10] F. Stein and G. Medioni, "Structural indexing: efficient 3-D object recognition", IEEE Transactions on 
Pattern Analysis and Machine Intelligence, vol. 14, no. 2, (1992), pp. 239-256.

[11] P. J. Besl and N. D. McKay, "A method for registration of 3D shapes", IEEE Transactions on Pattern Analysis and Machine Intelligence, vol. 14, no. 2, (1992), pp. 239-256.

[12] Y. Chen and G. Medioni, "Object modelling by registration of multiple range images, Image and Vision Computing", vol. 10, no. 3, (1992), pp. 145-155.

[13] D. Grant, J. Bethel and M. Crawford, "Point-to-plane registration of terrestrial laser scans", ISPRS Journal of Photogrammetry and Remote Sensing, vol. 72, (2012), pp. 16-26.

[14] Y. D. Eo, M. W. Pyeon, S. W. Kim, J. R. Kim and D. Y. Han, "Coregistration of terrestrial lidar points by adaptive scale-invariant feature transformation with constrained geometry", Automation in Construction, vol. 25, (2012), pp. 49-58.

[15] N. Senin, B. M. Colosimo and M. Pacella, "Point set augmentation through fitting for enhanced ICP registration of point clouds in multisensor coordinate metrology", Robotics and Computer-Integrated Manufacturing, vol. 29, no. 1, (2013), pp. 39-52.

[16] Y. J. Li, X. Y. Hu, J. Q. Zhang, W. S. Jiang and Y. J. Zhang, "Automatic road extraction in complex scenes based on information fusion from lidar data and remote sensing imagery", Acta Geodaetica et Cartographica Sinica, vol. 41, no. 6, (2012), pp. 870-876.

[17] X. H. Tang, T. L. Wang, Y. J. Jun, Z. K. Feng, D. Q. Liu, M. L. Lie and Y. Li, "Discussion on the principles of forest measurement in fixed sample plot with three Dimensional laser scanning aided with digital close range photogrammetry", Journal of Anhui Agri. Sci., vol. 38, no. 12, (2010), pp. 6095-6097.

[18] F. Deng, H. G. Yang and L. Y. Li, "Registration between lidar and laser image by using mutual information", Science of Surveying and Mapping, vol. 34, no. 6, (2009), pp. 51-52.

[19] F. Deng, Z. X. Zhang and J. Q. Zhang, "3D reconstruction of old architecture by laser scanner and digital camera", Science of Surveying and Mapping, vol. 32, no. 2, (2007), pp. 29-30.

\section{Authors}

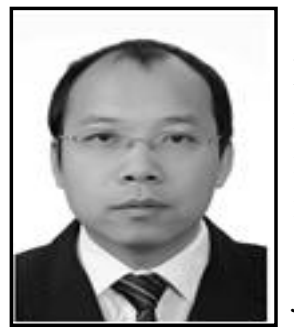

Wenshu Lin, he earned a B.E. degree and a M.S. degree in Forest Engineering from Northeast Forestry University, Harbin, P. R. China in 2003 and 2005, respectively. In 2011, he received a Ph.D. degree in Forest Resources Science from West Virginia University, USA. He is a Member of Forest Products Society and Society of Wood Science and Technology. Dr. Lin had several publications in esteemed journals such as Forest Products Journal, Wood and Fiber Science, and Computers and Electronics in Agriculture. Currently, he is an associate professor at Northeast Forestry University, Harbin, P. R. China. His major research interests include forest and environment remote sensing monitoring, computer modeling and $3 \mathrm{D}$ visualization, nondestructive testing and evaluation of standing trees and wood.

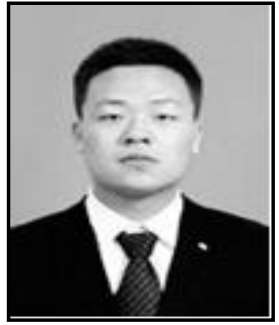

Yang Li, he earned a B.E. degree in Remote Sensing Science and Technology from Shandong Agriculture University, Shandong, P. R. China in 2015. Currently, he is a master student at Northeast Forestry University. His major research interests include forest and environment remote sensing monitoring, computer modeling and 3D visualization.

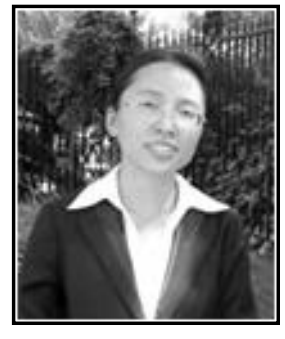

Jinzhuo Wu, she earned her B.E. degree and M.S. degree in Forest Engineering from Northeast Forestry University, Harbin, P. R. China in 2003 and 2005, respectively. In 2010, she obtained her $\mathrm{Ph} . \mathrm{D}$. degree in Forest Resources Science from West Virginia University, USA. She is a Member of Forest Products Society. Dr. $\mathrm{Wu}$ had several publications in esteemed journals such as Forest Products Journal, Wood and Fiber Science, Canadian Journal of Forest Research, and Journal of Agriculture and Resources Economics. 


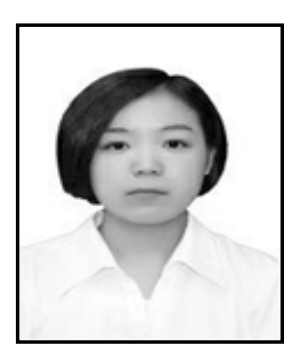

Shanshan Zhang, she earned a B.E. degree in Forest Engineering from Northeast Forestry University, Harbin, P. R. China in 2015. Currently, she is a master student at Northeast Forestry University. Her major research interests include forest and environment remote sensing monitoring, computer modeling and algorithm design.

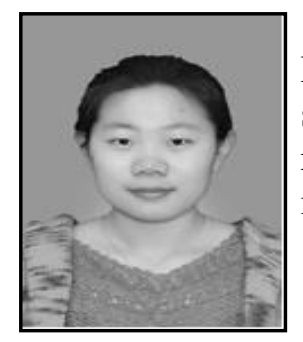

Yuan Meng, she earned a B.E. degree in Forest Engineering from Northeast Forestry University, Harbin, P. R. China in 2015. Currently, she is a master student at Northeast Forestry University. Her major research interests include forest and environment remote sensing monitoring, computer modeling and algorithm design. 